\title{
LE SORT DES INCITATIONS FISCALES APPLICABLES AUX INDUSTRIES EXTRACTIVES DANS LES CODES MINIERS CONGOLAIS (RDC) ET GUINEEN TELS QUE REVISES
}

\author{
Par DJANGI EKILA Nicole*
}

\section{Résumé}

Cet article fait une étude horizontale des incitations fiscales avant et après la révision des Codes miniers en RDC et en Guinée. Cette étude se veut être comparative et critique dans la perspective d'une meilleure gestion du secteur extractif et d'une maximisation des recettes des Etats sous examen. Ainsi de la présentation des politiques fiscales minières dans ces deux pays, passant par l'état de lieux des incitations dans les différents codes avant et après la modification pour aboutir aux critiques et perspectives, cette étude peint un tableau descriptif qui permette de comprendre ce qu'il y avait et ce qui a changé dans les différentes législations.

\begin{abstract}
This article is a horizontal analysis of fiscal incentives before and after the revision of the Mining Codes in DRC and Guinea. This study is intended to be comparative and critical from the perspective of better management of the extractive sector and maximization of revenue for the states under review. Thus, from the presentation of the mining tax policies in these two countries, through the state of play of incentives in the different codes before and after the modification, to the criticisms and perspectives, this study paints a descriptive table that allows an understanding of what was and what has changed in the different legislations.
\end{abstract}

\section{Introduction}

L'industrie extractive est définie comme étant « toute unité d'extraction, de transformation et de commercialisation de l'un ou l'autre produit minéral, pétrolier et forestier ${ }^{1} \gg$. Prise

* Nicole DJANGI EKILA est actuellement apprenante en troisième cycle pour l'obtention d'un diplôme d'études supérieures (DES) en Droit économique et social à la Faculté de droit de l'Université de Kinshasa (RDC), Avocate au Barreau près la Cour d'Appel de Kinshasa/Matete, Chercheure au CREEDA et LRT Alumni du Projet AMLA. Mes recherches portent sur la fiscalité dans le secteur minier, la gouvernance et la transparence dans la gestion des ressources tirées de ce secteur par les Etats.

1 ITIE-RDC, Synthèse du rapport. Exercice 2007, Kinshasa, 2010, p. 6. 
dans ce sens, l'industrie extractive englobe les entreprises minières, les comptoirs et les centres de négoces ${ }^{2}$. Dans le cadre de cet essai, l'industrie extractive se limitera à l'unité d'extraction, de transformation et de commercialisation des substances minérales notamment les métaux non-ferreux ${ }^{3}$, les métaux ferreux, les pierres précieuses ${ }^{4}$, les métaux précieux $^{5}$ et rares $^{6}$ faisant l'objet d'une exploitation industrielle ou à grande échelle ${ }^{7}$ par les entreprises minières.

Tous ces minerais, renfermés dans le sol et le sous-sol des pays colonisés, sont indispensables à l'évolution de la technologie de pointe ${ }^{8}$ et font l'objet depuis la fin du XIX ${ }^{\text {ème }}$ siècle d'une quête effrénée des pays dits industrialisés. L'accès à ces ressources est un enjeu majeur surtout pour les multinationales ${ }^{9}$ et les mécanismes d'accession oscillent entre les voies licites et l'instrumentalisation des guerres. La RDC en est un exemple.

Qualifiées de scandales géologiques, la $\mathrm{RDC}^{10}$ et la Guinée ${ }^{11}$ disposent d'une gamme aussi abondante que diversifiée des ressources minérales qui contraste avec l'état de la pau-

2 Joseph CIHUNDA HENGELELA, Quête permanente du cadre juridique approprié à l'industrie extractive en RDC. Quelques réflexions sur les logiques directrices de la reforme minière de 2002, KAS African Law Study Library, Kinshasa, 2014, p.1.

3 La Loi n ${ }^{\circ} 18 / 001$ du 9 mars 2018 modifiant et complétant la loi nº07/2002 portant Code minier congolais prévoit en son article $1^{e r}$ point 28septies : "les métaux non-ferreux sont métaux de base (cuivre, étain, aluminium, nickel, zinc et plomb) auxquels peuvent être ajoutés certains métaux rares et semi-précieux comme le titane, le cobalt, le vanadium et le molybdène. Ces métaux entrent dans la composition des alliages ne contenant que très peu ou pas de fer $》$.

4 Le code minier guinéen amendé considère comme pierre précieuse : la pierre gemme de haute valeur dont le diamant, le rubis, le saphir et l'émeraude alors que le législateur congolais, lui, met dans la catégorie des pierres précieuses : les substances minérales précieuses constituées d'un ou de plusieurs éléments chimiques et possédant les propriétés particulières qui leur donnent ainsi une valeur marchande élevée. Il s'agit de : diamant, émeraude, rubis, saphir, chrysobéryl et topaze, andésine, tanzanite, corindon, tourmaline et toute autre pierre de joaillerie de valeur comparable généralement négociée en carats.

5 Les métaux précieux sont : l’Argent, l’Or, les Platinoïdes, le Palladium et le Rhodium.

6 Les métaux rares sont : l'uranium, le tantale, la colombite, le wolfram, le béryllium, la monazite, la cassitérite ou minerai d'étain, le platine et le rhénium.

7 Il s'agit de toute exploitation dont les activités consistent à extraire et à concentrer des substances minières et à récupérer les produits marchands par des méthodes et procédés modernes et mécanisés.

8 CIHUNDA, Quête permanente du cadre juridique approprié à l'industrie extractive en RDC. Quelques réflexions sur les logiques directrices de la reforme minière de 2002, p.2.

9 CIHUNDA, Quête permanente du cadre juridique approprié à l'industrie extractive en RDC. Quelques réflexions sur les logiques directrices de la reforme minière de 2002, note 2.

10 La RDC est un pays dont le sol et le sous-sol recèlent une gamme aussi abondante que diversifiée des ressources naturelles notamment minières qui, ajoutée à l'immensité de son territoire, le place parmi les géants de l'Afrique après l'Algérie. Son potentiel minier est constitué des minerais suivant : le cobalt, le cuivre, l'or, l'argent, le charbon, l'aluminium, le niobium et tantale, le mercure, l'acier, le ciment, le diamant, le zinc, le phosphate, etc.

11 La Guinée est dotée des réserves de minéraux les plus convoités de la planète dont la plus grande réserve du monde de bauxite, le fer, le diamant, l'or et des quantités indéterminées d'uranium, lire 
vreté des populations de leurs pays. Le paradoxe de la pauvreté vécu par les populations congolaise $^{12}$ et guinéenne ne cesse d'interpeller les décideurs sur les transformations à mener pour l'élaboration de nouvelles politiques dans le secteur extractif en vue de garantir le développement.

Cette triste image des secteurs miniers congolais et guinéen est certainement due à la qualité de la gouvernance de cette industrie tant dans son aspect juridique qu'économique notamment fiscal. C'est ce dernier aspect qui constitue l'objectif de la présente réflexion. Il s'agit principalement de comprendre les logiques directrices des réformes minières dans leurs aspects fiscaux entre autres les incitations fiscales accordées aux opérateurs intervenues en RDC à partir de 2002 et en Guinée à partir de 2011 dans le secteur minier industriel.

Les législateurs du 11 juillet 2002 et du 09 septembre 2011 avaient considérés leurs œuvres comme celles qui allaient résoudre les problèmes d'attractivité des investisseurs, particulièrement étrangers et détenteurs de grands capitaux, ainsi que de maximisation des recettes tirées de l'exploitation des mines dans le produit intérieur brut.

Bénéficiant des expériences des législations antérieures, les codes miniers de 2002 et 2011 étaient conçus comme des instruments qui allaient attirer les investisseurs, accroître les revenus publics au budget des Etats et contribuer à la relance des économies. Ces deux législateurs caressaient le rêve pour l'un de voir le secteur minier redevenir le fleuron de l'économie congolaise comme dans les décennies 70 et $80^{13}$ ainsi que pour l'autre de voir ces actifs miniers profitaient à l'ensemble de la population plutôt qu'à quelques sociétés minières et politiciens internationaux sans scrupules ${ }^{14}$.

Seize ans après pour la RDC et deux ans après pour la Guinée, le bilan de la gouvernance du secteur minier n'a pas été à la hauteur des attentes dans ces deux Etats. Les législations minières, œuvres du législateur, n'échappant pas aux règles d'imperfections vont se révéler lacunaires à cause des manquements faisant échapper des gains aux Etats et du fait de la libéralisation à outrance. Les ressources perçues des activités minières n'étaient que trop faibles ${ }^{15}$ pour prétendre booster l'économie de ces pays ainsi que leur développement, sans oublier le bien-être social de leurs populations respectives.

attentivement https://www.un.org/africarenewal/fr/magazine/avril-2014/ressources-mini\%C3\%A $8 \mathrm{r}$ es-la-fin-d\%E2\%80\%99une-mal\%C3\%A9diction (accédé dernièrement le 23 octobre 2018 à 11h00).

12 Nicole DJANGI EKILA, L'Impact du Régime Fiscal applicable au Secteur Minier Industriel sur le Développement de la République Démocratique du Congo, Mémoire de Licence, UCC, Kinshasa, 2017, p.1.

13 CIHUNDA, Quête permanente du cadre juridique approprié à l'industrie extractive en RDC. Quelques réflexions sur les logiques directrices de la reforme minière de 2002, note 2, p.3.

$14 \mathrm{https} / /$ www.theguardian.com/global-development/poverty-matters/2013/jun/14/guinea-esource-w ealth-work-people (accédé dernièrement le 10 décembre 2018 à 19heures).

15 En effet dans le cas de la RDC, on note que la contribution du secteur minier dans le budget de l'Etat n'a varié qu'entre 9,40 et $21,75 \%$. Pour la Guinée, on note que la contribution a évolué entre 1,9 et 3,9 dans le PIB. 
C'est ainsi que la RDC et la Guinée vont se lancer dans le mouvement de révision de leurs codes miniers ${ }^{16}$ entamé par le gouvernement en collaboration avec la société civile avec comme objectif revoir les avantages excessifs accordés aux opérateurs miniers. Ce processus de révision des lois minières est une preuve attestant que la RDC et la Guinée sont encore en quête du cadre juridique approprié pour leur secteur minier, notamment en ce qui concerne la fiscalité applicable.

Cet article se structure autour de la question relative au sort des incitations fiscales dans l'exploitation industrielle des mines après la révision de ces codes miniers. Pour arriver à comprendre ce sort, nous avons emprunté une démarche qui associe la dogmatique juridique à la sociologie du droit dans une approche comparative et critique. Cette étude est essentiellement juridique faisant l'apologie du droit dans l'amélioration de la gouvernance des secteurs miniers congolais et guinéen et de leur contribution au développement.

La présente étude revêt un intérêt particulier dans le contexte actuel de la RDC et de la Guinée. Cet intérêt est de cinq ordres : premièrement, ces deux pays sont tous considérés comme des scandales géologiques en raison de la quantité des ressources qu'on y trouve mais aussi, de la variété et de la forte demande de ces minerais; deuxièmement, les ressources minières contenues dans le sol et le sous-sol sont de droit la propriété de ses Etats ${ }^{17}$, notamment de leurs citoyens ${ }^{18}$, leur exploitation par des particuliers doit en contrepartie permettre aux Etats d'assurer le développement de leurs populations; troisièmement, la RDC et la Guinée en dépit de leurs ressources minières sont des pays à risque pour l'investissement à cause du climat d'instabilité politique et civile qui y règnent; quatrièmement, les incitations fiscales sont la clé de voûte de toute législation minière attractive et ne doivent pas être utilisées comme un repoussoir d'investissements mais plutôt comme un aimant; et enfin, cinquièmement, la révision du Code minier congolais intervenue en mars dernier est sujette à plusieurs débats et crée encore des tensions entre l'Etat et les investisseurs.

Le présent essai comprendra trois points : le premier mettra en lumière les considérations de la politique fiscale minière en RDC et en Guinée $(\boldsymbol{A})$, le deuxième fera l'état des lieux des incitations fiscales applicables aux industries extractives dans ces deux pays $(\boldsymbol{B})$ et le troisième abordera les critiques et perspectives de ces révisions $(\boldsymbol{C})$.

16 La RDC et la Guinée ne sont pas les seuls pays à s'être lancé dans le mouvement de révision des codes miniers, lire attentivement, Hany BESADA et Philip MARTIN, Les codes miniers en Afrique : la montée d'une « quatrième» génération?, INS, Rapport de recherche, Mai 2013.

17 Article 3 des Lois $\mathrm{n}^{\circ} 007 / 2002$ du 11 juillet 2002 portant Code minier congolais et L/ 2011/006/CNT du 9 septembre 2011 portant Code minier de la République de Guinée.

18 Article 58 de la Constitution du 18 février 2006 telle que modifiée à ce jour, pour la RDC et; pour la Guinée, article 21 de la Constitution du 7 mai 2010. 


\section{A. Considérations générales de la politique fiscale minière en RDC et en Guinée}

La politique minière en général et, celle fiscale minière, en particulier en Afrique ont toujours été marquées par le besoin pour l'Etat de maximiser les recettes tirées de l'exploitation de ses ressources naturelles.

Ces politiques ont été consacrées dans les différentes législations en la matière dont il nous faut faire ici un bref historique partant de la libéralisation du secteur et de la libéralisation atténuée ${ }^{19}$ qui s'en est suivie pour la RDC (I) et la Guinée (II).

\section{Code minier de la République Démocratique du Congo}

Le secteur minier a toujours été considéré comme le levier du développement en RDC; et le législateur, depuis l'époque coloniale, a toujours été guidé, dans la mise en place de la politique fiscale minière, par un besoin de maximisation des recettes perçues par l'Etat à l'issue de l'exploitation industrielle des mines pour impulser le développement de sa population.

Dans cette quête des recettes par l'Etat, le législateur va en 2002 adopté la Loi n ${ }^{\circ} 007 / 2002$ du 11 juillet 2002 portant Code minier et son règlement d'application ${ }^{20}$. Cet instrument juridique, pris dans un contexte de crise économique sévère dans le secteur ${ }^{21}$ alors socle de l'économie congolaise, se voulait être incitatif et attractif dans le souci de faire revenir les investisseurs et de pallier aux insuffisances des précédentes législations.

Cette nouvelle législation, sur laquelle reposaient tous les espoirs du peuple congolais, présentait un caractère incitatif avec des procédures d'octroi des droits miniers ou de carrières objectives, rapides et transparentes dans laquelle sont organisés des régimes fiscaux, douaniers et de change ${ }^{22}$. Pour la première fois, un régime fiscal unique prévu dans le Code va s'appliquer à tous les opérateurs du secteur minier industriel, exception faite des opérateurs régis par des conventions avec l'Etat; cela avec comme principe directeur la maximisation des recettes de l'Etat en s'adaptant aux réalités, spécificités et particularités du secteur.

Le régime fiscal du Code de 2002 combinait les caractères exhaustif, exclusif, extensif et stable. Si ce régime a eu le mérite de faire revenir les investisseurs ${ }^{23}$; cependant, très vite, il va se faire sentir le besoin de modifier cet instrument juridique. Les raisons de la modifi-

19 Voir BESADA et MARTIN, Les codes miniers en Afrique : la montée d'une « quatrième» génération?, Op.cit.

20 Décret $n^{\circ} 038 / 2003$ du 26 mars 2003 portant Règlement minier, in JORDC, numéro spécial du 1 avril 2003.

21 Grégoire BAKANDEJA wa MPUNGU, Droit minier et des Hydrocarbures en Afrique Centrale: Pour une gestion rationnelle, formalisée et transparente des ressources naturelles, Bruxelles, 2009, p.95.

22 Exposé des motifs du Code minier de 2002, p.2.

23 En effet, un rapport du Sénat de 2009 renseigne qu'il y avait à la date de ce rapport 1130 opérateurs miniers personnes physiques et morales dont : 368 opérateurs miniers au Katanga, seulement 245 sont réellement exploitants miniers; 39 opérateurs miniers au Maniema, 22 au Nord-Kivu, 59 
cation ont été nombreuses, une particulièrement, a retenu notre attention notamment celle liée à la faible contribution des recettes tirées de l'exploitation minière dans le budget de l'Etat. Il a été constaté depuis 2012, que le pourcentage du rendement du secteur minier variait entre 9,40 et $21,75 \%$ dans le budget de l'Etat.

Après une première tentative de modification qui a échoué en $2012^{24}$; le débat sera repris en 2015 pour aboutir à une modification en 2018. Depuis 2018, le siège de la matière est la Loi n007/2002 du 11 juillet 2002 telle que modifiée et complétée par la Loi n 18/001 du 9 mars 2018 et son règlement d'application ${ }^{25}$.

L'adoption de ce texte, organisant actuellement la gestion du secteur minier dont la fiscalité minière applicable aux industries extractives est le fruit d'un long processus des consultations mouvementées. Il inaugure une nouvelle politique fiscale basée sur la révision des avantages ou incitations accordés aux opérateurs sous le Code de 2002, elle fera l'objet d'analyse au second point de cette étude. Ce qui nous permet de revisiter la politique fiscale minière telle que consacrée par le code guinéen.

\section{Code minier de la République de Guinée (Conakry)}

Le secteur minier industriel de la Guinée est actuellement régi par la Loi L/2011/006/CNT du 9 septembre 2011 portant Code minier partiellement amendée par la Loi L / 2013/ 053 / CNT du 8 avril 2013 portant amendements de certaines dispositions. A ces textes, il faut ajouter le Code minier communautaire de l'UEMOA du 23 décembre 2003 et la Directive minière de la CEDEAO C / DIR 3 / 05 / 09 du 27 mai 2009.

Tout comme la RDC, la Guinée est un scandale géologique. Pays de l'Afrique de l'Ouest, il est un grand producteur de la bauxite et de l'aluminium dont les réserves constituent le tiers de celles de toute la planète. A ce titre, le secteur minier a été placé au cœur d'un vaste et profond processus de réforme visant à renforcer son impact sur l'économie nationale, et plus généralement sur le développement socio-économique du pays ${ }^{26}$.

Les législations minières, baromètres de l'investissement dans les activités minières et moyen d'accroître les revenus de l'Etat, ont toujours fait l'objet de préoccupations premières du gouvernement. C'est ainsi qu'en 2011, la Guinée va adopter un nouveau Code minier après avoir conclu que le précédent, celui de $1995^{27}$, n'avait pas permis d'atteindre le développement tant espéré au moyen de la fiscalité applicable dans le secteur extractif.

au Sud-Kivu et 94 en Province Orientale. Dans les provinces de l'Ouest (Kinshasa, Bas-Congo, Bandundu et Equateur), la Commission avait répertorié 89 opérateurs miniers.

24 Dépôt de l'avant-projet de révision du Code minier de 2002 en 2015.

25 Décret $n^{\circ} 18 / 024$ du 08 juin 2018 portant Règlement minier, in JORDC, numéro spécial de juillet 2018.

26 http://invest.gov.gn/page/mines?onglet=presentation, (accédé dernièrement le 20 octobre 2018 à 13h50).

27 Il s'agit de la Loi L/95/036/CTRN du 30 juin 1995 portant Code minier de la République de Guinée. 
En effet, le Code de 1995, en dépit de bonnes dispositions qu'il pouvait renfermer, n'avait été appliqué qu'à moitié laissant les grandes sociétés minières en matière de fiscalité sous un régime conventionnel avec des exonérations exorbitantes qui s'appliqueraient pour de nombreuses années en plus de la non-application de certains taux d'imposition.

Bien que les recettes globales tirées de l'exploitation des mines ont représentées $21,94 \%$ des recettes de l'Etat entre 2000 et 2009 avec une forte tendance à la baisse à partir de $2006^{28}$, ce sont des mouvements sociaux et des grèves prolongées dans certaines villes minières du pays suite à la hausse mondiale des prix des ressources minières combinée à la chute de la demande mondiale des matières premières qui vont contribué au changement à partir de $2007^{29}$.

Ces différents aspects, accentués par l'élection d'un nouveau gouvernement en 2010, vont être à la base d'un changement de cap avec l'adoption d'un nouveau code minier en 2011 concrétisant les mouvements sociaux qui ont touché la Guinée depuis 2007.

Cette nouvelle législation encadrant à la fois le régime conventionnel que celui de droit commun sous une même politique fiscale applicable aux industries extractives est vu comme donnant un nouveau souffle au secteur minier. Il se veut être attractif et incitatif.

Cependant, deux années après son application, soit en 2013, le Code va être partiellement amendé avec entre autres objectifs en ce qui concerne la fiscalité : favoriser plus de rentabilité pour les investisseurs et élargir la base des recettes fiscales du pays. Tout ceci s'inscrit dans une stratégie visant à faire des mines la locomotive du développement socioéconomique de la Guinée ${ }^{30}$.

Au regard de tout ce qui vient d'être dit sur la RDC et la Guinée, on peut constater que la politique fiscale minière de ces deux pays est essentiellement axée sur les incitations fiscales en faveur des industries extractives et les modifications en Afrique en générale et dans ces deux pays en particulier touchent essentiellement lesdites incitations, quid de l'état de lieux de ces allègements.

\section{B. Etat des lieux des incitations fiscales applicables aux industries extractives}

Les incitations fiscales sont des dispositions qui offrent des avantages financiers ou fiscaux aux titulaires d'un permis, dans le cas d'espèce un permis d'exploitation industrielle, pour faire de l'exploitation minière un investissement plus attractif ${ }^{31}$.

$28 \mathrm{https}$ ://www.guineepolitique.com/guinee-lapport-du-secteur-minier-au-developpement-economiqu e/, (accédé dernièrement le 27 novembre 2018 à $18 \mathrm{~h} 00$ ).

29 https://journals.openedition.org/ecgogeo/14758, (accédé dernièrement le 27 novembre 2018 à 19h00).

30 http://invest.gov.gn/page/mines?onglet=presentation, (accédé dernièrement le 20 octobre 2018 à 13h50).

31 AMLA, Manuel des Consignes relatives au Traitement et à l'Analyse des Renseignements utiles pour la plate-forme, Document préparé par Nneoma Veronica Nwogu, version 2017, p. 51. 
Ces allègements fiscaux, accordés par l'Etat aux investisseurs, sont élaborés pour contrebalancer les éventuels aléas pouvant subvenir pendant la durée de l'investissement minier ${ }^{32}$. Ils tiennent compte de l'importance des investissements dans le secteur, du temps important que prend un projet minier avant d'être rentable, du flux des prix des matières premières sur le marché dont les variations ne peuvent être anticipées et du climat civile et politique au sein de l'Etat d'accueil.

Il est important de souligner que le contexte africain en général, et celui congolais et guinéen en particulier ${ }^{33}$, a beaucoup influencé le législateur dans la détermination des avantages fiscaux à accorder aux investisseurs dans les différents codes. En effet, en dépit de sa géographie particulière, l'Afrique demeure un continent instable sur les plans politique et civil; cette instabilité, qui le désavantage, ne lui permet pas de garantir un environnement sans risque aux opérateurs.

En règle générale, on inclut dans les incitations fiscales les dispositions relatives à la réduction ou l'exonération d'impôts et taxes ${ }^{34}$, celles visant à déduire certaines dépenses de l'assiette du bénéfice imposable ${ }^{35}$, celles relatives aux règles d'amortissement ${ }^{36}$ ainsi que celles qui concernent la clause de stabilisation ${ }^{37}$.

Pour comprendre l'état de lieux des incitations fiscales minières, nous donnerons d'abord les incitations fiscales qui existaient dans les Codes miniers congolais et guinéen avant la révision (I) et ensuite, le sort de ces allègements fiscaux après la révision (II).

\section{Incitations fiscales prévues dans les Codes miniers avant la révision}

Avant la révision des Codes miniers de la RDC et de la Guinée, les législateurs prévoyaient les incitations fiscales suivantes, selon les différentes catégories :

32 Tracy MBUYU KABWE, Le mouvement de révision des codes miniers en Afrique: l'exemple de la République Démocratique du Congo, Mémoire de Master, Paris, 2014, p.13.

33 En 2002 lorsque le Code minier de la RDC voyait le jour, le pays sortait d'une crise politique importante avec l'assassinat de son président en 2001 sans compter les multiples rébellions existantes dans l'arrière-pays, principalement dans les régions minières, organisées par différents groupes armés. De même pour la Guinée, en 2011, qui sortait d'une crise civile tempérée par l'élection présidentielle.

34 Il s'agit des dispositions qui tendent à long ou à court terme à soit imposer à un faible taux en comparaison au taux applicable dans la fiscalité de droit commun, soit à soustraire totalement le contribuable au paiement d'un certain nombre d'impôts ou taxes.

35 Il est question des certaines dépenses que le législateur entend soustraire de l'assiette de l'impôt généralement de l'impôt sur les bénéfices.

36 Ces règles visent la manière dont le législateur entend permettre au titulaire d'un permis industriel de recouvrer les dépenses engagées dans la construction et à quelle durée.

37 La classification avec les trois premiers éléments est celle que nous tirons du Manuel d'AMLA à l'exception de la clause de stabilisation que nous avons inclut parce que nous considérons qu'elle fait partie des incitations fiscales ayant pour de garantir les avantages acquis par les investisseurs. 


\section{La réduction ou l'exonération d'impôts et taxes}

En rapport avec nos deux codes miniers en étude, il est possible de relever que ce soit avant ou après la modification, le législateur congolais a adopté un système de réduction et exonération à long terme ${ }^{38}$ contrairement, à celui guinéen ${ }^{39}$ qui a préféré le système à court terme.

Le Code minier congolais de 2002 prévoyait que le titulaire du permis d'exploitation industriel soit exonéré de : l'impôt foncier pour les immeubles situés à l'intérieur de la superficie des concessions minières et assujettis à l'impôt y afférent ${ }^{40}$, l'impôt sur les véhicules de transport des personnes ou des matériaux, de manutention ou de traction et de la taxe spéciale de circulation routière lorsqu'ils sont utilisés exclusivement dans l'enceinte du projet minier ${ }^{41}$, l'exemption à l'impôt mobilier des intérêts payés par le titulaire en vertu des emprunts contractés en devise à l'étranger sous réserve du respect des conditions requises quant à $\mathrm{ce}^{42}$ et la TVA à l'intérieur sur les produits vendus à une entité de transformation située sur le territoire national ${ }^{43}$.

A ces exonérations, le législateur avait prévu des réductions des taux des impôts suivants : l'impôt sur la superficie des concessions minières et hydrocarbures (pour le permis d'exploitation $0,0481 \$$ et pour le permis d'exploitation des rejets $0,0481 \$$ après ajustement des montants par le CAMI en 2008), l'impôt mobilier sur les dividendes et autres distributions versés par le titulaire au taux de 10\%, l'impôt professionnel sur les bénéfices au taux de $30 \%$, l'impôt professionnel sur les rémunérations des expatriés au taux de $10 \%$ et la TVA à l'intérieur à des taux différents dépendant des circonstances de 10,5 et $3 \%$.

Par contre pour la Guinée, il faut noter deux aspects importants : d'abord les avantages fiscaux sont repartis selon les différentes phases d'évolution du projet minier. Ainsi, en phase de construction, l'opérateur bénéficie des exonérations suivantes : la Taxe sur la Valeur Ajoutée (TVA) sur les importations des équipements, matériels, machines, matières premières et consommables visés par la liste minière soumise, avant le démarrage de la phase de construction, sous réserve que cette liste minière ait été agréée conformément aux dispositions de l'article 166 du présent code; l'Impôt Minimum Forfaitaire (IMF); la Contri-

38 Ainsi par exemple, lorsque le législateur congolais prévoit que l'impôt exceptionnel sur la rémunération des expatriés soit payé au taux de $10 \%$, il ne fait pas de différence entre les différentes phases du projet minier; alors que, le législateur guinéen fait une différente par exemple entre l'exonération de la TVA en phase de recherche, de construction et d'exploitation.

39 Il est possible de relever que ces incitations varient selon qu'on se trouve dans la phase d'installation ou de construction et selon que l'opérateur commence l'exploitation.

40 Articles 236 du Code minier de 2002 et 521 du Règlement minier de 2003.

41 Articles 237 et 239 du Code minier de 2002 ainsi que 522 du Règlement minier de 2003.

42 Articles 528 à 533 du Règlement minier de 2003.

43 Articles 534 à 536 du Règlement minier de 2003. 
bution des patentes; la Contribution à la formation professionnelle; la Contribution Foncière Unique (CFU); et de la Taxe d'apprentissage ${ }^{44}$.

En phase d'exploitation, le titulaire bénéficie pendant trois (3) ans de l'exonération des impôts notamment de de l'Impôt Minimum Forfaitaire (IMF) et de la Contribution Foncière Unique, la Contribution à la formation professionnelle et la Taxe d'apprentissage ${ }^{45}$.

\section{La déduction de certaines dépenses à l'assiette}

Les éléments pouvant être déduit de l'assiette de calcul de l'impôt sur les bénéfices sont limitativement énumérés par le législateur ${ }^{46}$ de même que les différentes provisions pouvant être constituées en franchise d'impôt ${ }^{47}$ dont : la provision pour réhabilitation des sites miniers, la provision pour reconstitution de gisements et en plus pour la Guinée des contributions au Fonds de Développement Local et des pertes de change enregistrées à la suite de fluctuations du cours des changes selon les modalités définies dans le Code Général des Impôts.

\section{Les règles d'amortissement}

En RDC, le code de 2002 prévoyait un amortissement dégressif avec option de différer en période déficitaire sans limitation du temps sur les exercices subséquents ${ }^{48}$. La Guinée, quant à elle, favorisait l'amortissement dégressif ${ }^{49}$.

\section{La clause de stabilisation}

La mise en œuvre de cette clause ainsi que son application diffère d'un pays à l'autre; tantôt, elle sera comptée à dater de l'octroi du titre ${ }^{50}$; tantôt à partir de la modification du code notamment de sa fiscalité 51 .

44 Article 173 du Code minier de 2011.

45 Article 175 du Code minier de 2011.

46 Pour la RDC lire les articles 243, 251, 254 et 255 du Code minier de 2002. Par contre, pour la Guinée, consulter utilement l'article 177 du Code de 2011.

47 En ce concerne la RDC, lire les articles 257 et 258 du Code minier de 2002. Pour le cas de la Guinée, voir l'article 178 du Code de 2011.

48 Articles 249 et 250 du Code de 2002.

49 Article 177 alinéa 1 tiret 5 du Code de 2011.

50 C'est le cas de la politique fiscale minière de la Guinée, voir Article 182 du Code de 2011 qui prévoit une période de 10ans de stabilisation du régime pour 1'exploitant industriel avec option d'extension de 5ans contre prime annuelle.

51 Tel est le cas de la vision de la fiscalité minière de la RDC, consulter l'article 276 du Code de 2002 qui consacrait une période de stabilisation de 10ans à compter de la modification du Code et surtout de ces aspects fiscaux, douaniers et de change. 
Après avoir passé en revue les incitations fiscales prévues par les législateurs avant la révision, il est important d'aborder le sort de ces incitations.

\section{Sort des allègements fiscaux après les Codes miniers révisés}

Le Code minier de la Guinée a été révisé en 2013, soit 2ans après son adoption et celui de la RDC, 16 ans après. Quelles sont les modifications apportées par les législateurs en ce qui concernent les incitations fiscales applicables aux industries extractives?

Il est possible de relever qu'en ce qui concerne la réduction ou l'exonération d'impôts et taxes, le législateur congolais de 2018 a conservé la majorité des exonérations à l'exception de la TVA dont le taux a été ramené au même pourcentage que celui de droit com$\operatorname{mun}^{52}$ (soit 16\%) et de l'impôt sur les rémunérations des expatriés qui sera désormais payé à $50 \%$ du taux de droit commun pendant $10 \mathrm{ans}$ (soit 12,5\%) et au taux de droit commun après 10 ans (soit $25 \%)^{53}$.

Quant au législateur guinéen, on remarque que pendant la phase de construction, il a été supprimé de l'exonération : l'impôt sur les sociétés, les droits d'enregistrement et des timbres ainsi que l'impôt sur les revenus des valeurs mobilières. A cela s'ajoute la suppression pendant la phase d'exploitation de la contribution à la formation professionnelle et de la taxe d'apprentissage pendant les trois premières années à compter de la première production. Quant à la contribution foncière unique, bien qu'elle soit conservée, son taux a été réduit à $10 \%$ pendant cette phase. Passé ce délai, le législateur prendra les dispositions pour son paiement pour le reste du temps.

En rapport avec la déduction de certains éléments de l'assiette de l'impôt sur les bénéfices ou impôt de sociétés, on constate que les deux lois minières suppriment certains éléments de la déduction ${ }^{54}$. L'amortissement, lui, n'a pas fait l'objet de révision en Guinée mais, en RDC, l'amortissement dégressif a été supprimé au profit de celui linéaire ${ }^{55}$.

Par ailleurs, la clause de stabilisation dans ces deux Etats a fait l'objet de révision. Si le législateur du Code minier de la Guinée est allé dans le sens d'augmenter la durée de cette mesure à $15 \mathrm{ans}^{56}$ à compter de l'octroi du titre minier et à supprimer l'option d'extension contre prime annuelle; celui de la RDC l'a plutôt réduit à $5 \mathrm{ans}^{57}$ à compter de l'octroi dudit titre.

Le présent tableau qui vient d'être fait sur le sort des incitations fiscales peut laisser croire que la révision des Codes miniers en 2013 pour la Guinée et en 2018 pour la RDC n'a été que bégnine en ce qui concerne les allègements fiscaux accordés aux opérateurs

52 Article 259 de loi de 2018.

53 Article 244 bis de la loi de 2018.

54 Article 177 de l'amendement au Code miner de la Guinée de 2013 et Article 256 al.1er litteras a, $c$, e et $i$; al. 2 litteras $a, b$, c et d et al. 3 de la loi modificative du Code minier congolais de 2018.

55 Article 9 de l'amendement de 2018.

56 Article 182 de l'amendement de 2013 de la Guinée.

57 Article 10 de l'amendement de 2018 de la RDC. 
dans le secteur industriel cependant, il est important de souligner que ces modifications ont élargi l'assiette fiscale en général dans le partage de la rente minière entre l'Etat et l'investisseur. Ceci nous pousse à aborder les critiques faites aux lois minières révisés ainsi que les perspectives pour une meilleure gestion du secteur minier dans ces pays.

\section{Critiques et Perspectives des révisions des Codes miniers congolais et guinéen}

Les Codes miniers de 2002 de la RDC et de 2011 de la Guinée sont issus de la libéralisation, voire de l'hyper-libéralisation du secteur visant à attirer les investisseurs. Au cours de leur application, l'insatisfaction de la part des Etats de ce régime libérationniste a conduit à leur révision pour des amendements atténuants l'hyper-libéralisation.

Ces amendements, ouvrant l'ère des révisions des codes miniers dans beaucoup de pays africains, ont eu pour objectifs de revoir la majeure partie des avantages accordés aux opérateurs en les augmentant ou en les supprimant totalement et pour l'occasion à créer d'autres impôts, taxes et droits.

Amendées, la plupart des incitations fiscales ne font plus partie des allègements fiscaux, mais plutôt des mesures fiscales prévues par le législateur et qui s'appliquent au secteur minier industriel. Cependant, la révision de ces codes appellent quelques critiques (I) ainsi que la proposition des perspectives (II) pour une meilleure gestion et applicable des dispositions issues de la modification.

\section{Critiques}

Les critiques ou points de fragilité des législations minières après leur révision seront abordés en deux temps : en premier lieu, il s'agira de relever les critiques intrinsèques aux lois minières c'est-à-dire les points de fragilité liés à la rédaction des textes et; en second lieu, les critiques extrinsèques ou celles liées à l'application des législations minières (dans le cas d'espèces en RDC et en Guinée).

Du point de vue intrinsèque, on peut soulever les critiques suivantes : primo, les incitations fiscales révisées ont augmenté la charge fiscale générale de l'opérateur minier industriel en élargissant l'assiette fiscale ${ }^{58}$. Si cet état de chose avait été conservé tel quel, il n'y aurait pas des mécontentements de la part des opérateurs. Cependant, l'on peut remarquer que les deux législateurs ne se sont pas contenté de supprimer les allègements fiscaux, ils ont en plus augmenté le taux de certains impôts, taxes et droits ${ }^{59}$ et plus loin pour la RDC

58 NRGI, La fiscalité du nouveau code minier de la République Démocratique du Congo, Analyse de Novembre 2018, p.2.

59 Pour la RDC, on peut relever que le législateur avec la modification a augmenté le taux de la redevance minière la participation gratuite de l'Etat à $15 \%$ soit $10 \%$ à l'octroi et $5 \%$ à chaque renouvellement. 
par exemple, le législateur a créé de nouvelles impositions ${ }^{60}$ bien que le législateur prévoit d'accorder des mesures incitatives de façon dérogatoire par voix d'arrêté du ministre ayant les mines dans ses attributions, cela ne résout pas le problème au contraire, il en crée un autre.

Secundo, dans le cas de l'amortissement, le législateur congolais qui opte pour l'amortissement linéaire perd de vue que le régime fiscal du Code minier est spécial, en vertu du principe « specialia generalibus derogant », dérogeant ainsi à celui de droit commun, il doit être plus avantageux que ce dernier. Or, avec la modification, il devient moins avantageux que celui de droit commun qui reste dans le contexte de l'amortissement dégressif.

Tertio, en ce qui concerne la clause de stabilisation dans les amendements de 2018 en RDC, sa rédaction est peu heureuse et suscite encore débat sur son application. En effet en 2002, cette clause prévoyait que les avantages accordés aux opérateurs soient conservés dix ans après si les dispositions fiscales et douanières du code étaient modifiées. En 2018, lors de la modification, le législateur, modifiant cette même clause non seulement réduit son délai d'application à cinq ans à dater de l'octroi du titre; est allé jusqu'à prévoir que la clause de stabilisation telle que prévue avant la modification ne pouvait plus s'appliquer après celle-ci au profit de la nouvelle disposition ${ }^{61}$. Cette violation flagrante de la loi a fait couler beaucoup d'encres, mais, pas celles du législateur qui jusqu'à ce jour n'a pas pris position pour se rétracter.

Du point de vue extrinsèque, les problèmes restent encore nombreux dans l'application des dispositions des lois minières surtout fiscales par les organes qui en ont la charge notamment l'administration fiscale.

Sur cet aspect la RDC et la Guinée se rencontrent; l'administration fiscale de ces deux pays connaissant les mêmes difficultés. Faisant suite aux critiques intrinsèques, il est possible de relever que : la fiscalité minière des Codes congolais et guinéen a toujours été difficile à appliquer par l'administration fiscale parce que combinant plusieurs aspects et est ainsi vue comme un monstre à plusieurs têtes. Cette difficulté est à la base de la non-collecte de certains impôts et du manque de contrôle a posteriori des opérateurs du secteur extractif. Les différentes révisions n'ont malheureusement pas tenu compte de cet aspect. Un vide criant en ce qui concerne la formation du personnel de l'administration fiscale sur l'application des dispositions financières des lois minières demeurent.

Une autre critique, pour les deux Etats, concerne le taux élevé de la corruption dans tous les domaines, et particulièrement pour cet essai, dans le secteur minier qui est une des situations les plus alarmantes. Dans ce contexte pour la RDC, par exemple, prévoir des inci-

60 La modification du Code minier de la RDC crée les impositions suivantes : l'impôt sur les profits excédentaires, le pas de porte, l'impôt professionnel des prestations sur les services, la taxe sur l'autorisation de minage temporaire, le droit d'octroi de la carte de travail pour étranger, les Taxes sur la télécommunication, la Taxe d'agrément des dépôts des Explosifs, le droit d'enregistrement des Dragues, etc. Lire utilement, le rapport du NRGI, La fiscalité du nouveau code minier de la République Démocratique du Congo.

61 Article 342 bis de la loi de 2018. 
tations par voie d'arrêté ministériel est une brèche ouverte par le législateur à plus de corruption mais surtout à des traitements inégaux des opérateurs dépendant de la volonté de l'organe qui en a la charge.

D'autres critiques comme le manque d'information des régies financières et les salaires insignifiants des agents de l'administration constituent encore là des fléaux à la gestion du secteur minier et des revenus que les Etats doivent percevoir de l'exploitation minière.

\section{Perspectives}

Les mesures fiscales sont la clé de voûte de toute législation minière attractive. Elles ne doivent pas être utilisées comme un repoussoir d'investissements, mais plutôt comme un aimant. Il ressort de l'étude des incitations fiscales que l'ère de révision inaugure une période d'amendement des codes miniers visant à réduire les avantages accordés au départ aux investisseurs ou à les supprimer dans le but de maximiser les recettes perçues par les Etats.

La révision intervenue en RDC en 2018, revisitant les incitations fiscales, les a vidés de leur contenu sans considération de certains aspects important dont le climat politique et civil du pays. Jusqu'ici si depuis 2002 le secteur à garder une contribution dans le budget de l'Etat en dépit du fait qu'elle soit considérée comme faible tenant compte des potentialités de ce pays, c'est notamment à cause du boom qu'il y a eu dans le secteur au début de la décennie 2000 et les discussions qui avaient débuté en 2012 marque la fin de ce boom.

En réalité, les différents régimes n'avaient pas empêché l'atteinte des objectifs mais, n'ont pas permis cela surtout à cause de raisons extérieures à ces lois. Il est donc important que la RDC et la Guinée résolvent les problèmes de la formation adéquate et continue du personnel des administrations fiscales sur l'application de la fiscalité minière industrielle, celle-ci ayant des facettes multiples; l'informatisation de l'administration; le contrôle a posteriori des opérateurs, le système étant déclaratif, très souvent l'administration ne procède pas au contrôle de véracité; dans le cas de la RDC le problème de la difficulté pour les membres de l'administration de procéder au recouvrement à l'intérieur du pays, les industries minières se situant souvent dans l'arrière-pays où les réseaux de communication n' existent pas ou sont en mauvais état, les membres de l'administration sont souvent obligés de parcourir plusieurs kilomètres à pied pour atteindre ses opérateurs. A cela s'ajoute le problème de la corruption, un fléau qui ne cesse de gangrener les pays africains en général, et la RDC ainsi que la Guinée.

Enfin, pour le cas particulier de la RDC, en ce qui concerne l'application de l'amortissement, que l'Etat privilégie l'amortissement dégressif comme prévu en 2002 au lieu de celui linéaire et pour la clause de stabilisation, qu'il s'engage à reconduire pour les opérateurs sous le régime de 2002 ce qui y était prévu et pour ceux à dater de 2018 du régime de la modification. 


\section{Conclusion}

La fiscalité minière est celle qui combine les intérêts a priori divergents d'attractivité des investisseurs et de maximisation des recettes par l'Etat ${ }^{62}$. Compris dans ce sens, la fiscalité minière apparaît comme étant la charpente de toute législation minière attractive. Utilisée correctement, elle permet d'attirer les investisseurs dans ce secteur à haut risque et d'augmenter les recettes de l'Etat.

Il ressort de l'étude des incitations fiscales dans les codes miniers révisés que la RDC comme la Guinée lors de l'adoption des Codes de 2002 et 2011 ont considéré que les régimes préexistants ne leur avaient permis de maximiser les recettes de leurs Etats; et dans les deux cas, il a été constaté que ces législations viennent après des moments de crise dans le secteur. Ces deux pays attendaient donc énormément des lois prises dans le domaine minier de par leur nature de scandale géologique.

Quelques années après l'application de ces codes, l'insatisfaction des gouvernements qui n'avaient pas assez bénéficié de l'augmentation des recettes va conduire aux révisions des Codes. Les législateurs vont mettre en avant le fait que si les législations ont permis d'attirer les investisseurs comme voulus par eux, l'accumulation des ressources par les Etats n'a pas suivi le pas et que cela était due aux avantages trop larges accordés aux opérateurs.

Ainsi, ils vont entamer la révisitation de ces avantages à travers la révision des Codes. Cependant, cette opération serait vaine si les différents Etats ne pensent pas à améliorer ou faciliter le travail de l'administration fiscale en se penchant sur les causes extrinsèques de fragilité des différentes législations minières dans leurs dispositions fiscales.

S'il était opportun de modifier les différentes lois, la façon d'y arriver n'a pas fait l'unanimité. Il est important que les Etats (RDC et la Guinée) ne perdent pas de vue la situation particulière de leurs pays où il est difficile d'investir mais aussi, d'engager toujours des débats francs avec toutes les parties prenantes pour ne pas être buté à un bras de fer avec les opérateurs.

Retenons que les incitations fiscales ne sont pas mauvaises dans les lois minières mais, lorsqu'elles sont organisées, elles doivent être prises de façon équitable pour un partage adéquat de la rente minière entre l'opérateur et l'Etat et; s'il s'avère que l'Etat entend les changer, il doit inviter toutes les parties à participer aux consultations afin de donner leurs avis.

62 DJANGI, L'Impact du Régime Fiscal applicable au Secteur Minier Industriel sur le Développement de la République Démocratique du Congo, p.40. 


\section{Bibliographie}

\section{A. TEXTES JURIDIQUES}

\section{Textes Constitutionnels}

Constitution du 18 février 2006 telle que modifiée par la Loi n 11/002 du 20 janvier 2011, in JORDC, $54^{\text {ème }}$ année, numéro spécial du 5 février 2011.

Constitution du 7 mai 2010 de la République de Guinée.

\section{Lois}

Loi n ${ }^{\circ} 007 / 2002$ du 11 juillet 2002 portant Code minier, in JORDC, $43^{\text {ème }}$ année, numéro spécial du 15 juillet 2002.

Loi L/2011/006/CNT du 9 septembre 2011 portant Code minier.

Loi $n^{\circ} 18 / 001$ du 9 mars 2018 modifiant et complétant la Loi n007/2002 du 11 juillet 2002 portant Code minier, $59^{\text {ème }}$ année, numéro spécial du 28 mars 2018.

Loi L / 2013/ 053 / CNT du 8 avril 2013 portant amendements de la Loi L/2011/006/CNT portant Code minier.

Avant-projet de révision du Code minier de 2002 en 2015.

\section{Règlements}

Décret n038/2003 du 26 mars 2003 portant Règlement minier, in JORDC, 44 ${ }^{\text {ème }}$ année, numéro spécial du 1 avril 2003.

Décret $n^{\circ} 18 / 024$ du 08 juin 2018 portant Règlement minier, in JORDC, $59^{\text {ème }}$ année, numéro spécial de juillet 2018.

\section{B. RAPPORTS ET COMMUNICATIONS}

AMLA, Manuel des Consignes relatives au Traitement et à l'Analyse des Renseignements utiles pour la plate-forme, Document préparé par Nneoma Veronica Nwogu, version 2017.

BESADA H. et MARTIN P., Les Codes miniers en Afrique : la montée d'une quatrième génération?, Rapport de recherche de l'Institut Nord-Sud, mai 2013.

CIHUNDA HENGELELA J., "Quête permanente du cadre juridique approprié à l'industrie extractive en RDC. Quelques réflexions sur les logiques directrices de la reforme minière de 2002 ", KAS African Law Study Library, 2014.

DJANGI EKILA N., L'Impact du Régime Fiscal applicable au Secteur minier Industriel sur le Développement de la République Démocratique du Congo, Mémoire de Licence, UCC, Kinshasa, 2017. ITIE-RDC, Synthèse du rapport. Exercice 2007, Kinshasa, 2010.

MBUYU KABWE T., Le Mouvement de Révision des Codes miniers en Afrique: L'exemple de la République Démocratique du Congo, Mémoire de Master, Paris, 2014. 
NRGI, La fiscalité du nouveau code minier de la République Démocratique du Congo, Analyse de Novembre 2018.

\section{WEBOGRAPHIE}

http://invest.gov.gn/page/mines?onglet=presentation.

https://www.un.org/africarenewal/fr/magazine/avril-2014/ressources-mini\%C3\%A8res-la-fin-d\%E2\%8

0\%99une-mal\%C3\%A9diction

https://www.guineepolitique.com/guinee-lapport-du-secteur-minier-au-developpement-economique/.

https://www.researchgate.net/publication/278825483_La_fiscalite_miniere_en_Afrique_de_l'Ouest_et _du_Centre.

https://www.researchgate.net/publication/278825483_La_fiscalite_miniere_en_Afrique_de_l'Ouest_et _du_Centre.

https://www.un.org/africarenewal/fr/magazine/avril-2014/ressources-mini\%C3\%A8res-la-fin-d\%E2\%8

0\%99une-mal\%C3\%A9diction.

https://www.theguardian.com/global-development/poverty-matters/2013/jun/14/guinea-esource-wealth -work-people. https://journals.openedition.org/ecgogeo/14758.

\section{Annexe: Tableau récapitulatif des incitations fiscales des Codes miniers Congolais (RDC) et Guinée. Sur ce tableau ne figure pas les éléments déductibles de la base d'imposition de l'impôt sur les bénéfices ou impôt de sociétés ainsi que les différentes provisions pour des raisons purement techniques.}

\begin{tabular}{|l|l|l|}
\hline \multicolumn{2}{|c|}{ Les Incitations Fiscales avant la Révision } & \multicolumn{1}{c|}{ Les Incitations Fiscales après la Révision } \\
\hline \multicolumn{2}{|c|}{ Code Minier Congolais de 2002 } & Code Minier Congolais révisé de 2018 \\
\hline Impôt sur les Bénéfices & $30 \%$ & $30 \%$ \\
\hline $\begin{array}{l}\text { Report des pertes (an- } \\
\text { nées) }\end{array}$ & 5 (sauf amortissement) & 5 \\
\hline $\begin{array}{l}\text { Report des pertes (li- } \\
\text { mite) }\end{array}$ & $100 \%$ & $100 \%$ \\
\hline Amortissement & $\begin{array}{l}\text { Exceptionnel de } 60 \% \\
\text { pour la première année. } \\
\text { Pour le reste des an- } \\
\text { nées, amortissement } \\
\text { dégressif. }\end{array}$ & Linéaire \\
\hline Impôt mobilier & $\begin{array}{l}10 \% \text { sur les divi- } \\
\text { dendes et } 0 \% \text { sur les } \\
\text { intérêts }\end{array}$ & $\begin{array}{l}10 \% \text { sur les dividendes et } 0 \% \text { sur les inté- } \\
\text { rêts. }\end{array}$ \\
\hline TVA & $10 \%$ & $16 \%$ soit taux de droit commun \\
\hline
\end{tabular}




\begin{tabular}{|c|c|c|}
\hline $\begin{array}{l}\text { Impôt exceptionnel sur } \\
\text { les } \\
\text { rémunérations versées } \\
\text { au } \\
\text { personnel expatrié. }\end{array}$ & $10 \%$ & $\begin{array}{l}50 \% \text { du taux de droit commun pendant } 10 \text { ans } \\
\text { (soit } 12,5 \% \text { ) et après au taux de droit com- } \\
\text { mun (soit } 25 \% \text { ), }\end{array}$ \\
\hline $\begin{array}{l}\text { Impôt sur les } \\
\text { véhicules }\end{array}$ & $\begin{array}{l}\text { N'est pas dû pour les } \\
\text { véhicules utilisés dans } \\
\text { l'enceinte de la conces- } \\
\text { sion minière. }\end{array}$ & $\begin{array}{l}\text { N'est pas dû pour les véhicules utilisés dans } \\
\text { l'enceinte de la concession minière. }\end{array}$ \\
\hline $\begin{array}{l}\text { Taxe spéciale de circu- } \\
\text { lation } \\
\text { routière }\end{array}$ & $\begin{array}{l}\text { N'est pas dû pour les } \\
\text { véhicules utilisés dans } \\
\text { l'enceinte de la conces- } \\
\text { sion minière. }\end{array}$ & $\begin{array}{l}\text { N'est pas dû pour les véhicules utilisés dans } \\
\text { l'enceinte de la concession minière. }\end{array}$ \\
\hline Impôt foncier & $\begin{array}{l}\text { N'est pas dû à l'inté- } \\
\text { rieur de la concession } \\
\text { minière }\end{array}$ & $\begin{array}{l}\text { N'est pas dû à l'intérieur de la concession mi- } \\
\text { nière. }\end{array}$ \\
\hline $\begin{array}{l}\text { Stabilisation de la légis- } \\
\text { lation }\end{array}$ & $\begin{array}{l}10 \text { ans après tout } \\
\text { amendement } \\
\text { du Code. }\end{array}$ & $\begin{array}{l}5 \text { ans à dater de l'octroi et après l'entrée en vi- } \\
\text { gueur du } \\
\text { code } 2018 \text { (pour les opérateurs ayant obtenu } \\
\text { le titre avant 2002) }\end{array}$ \\
\hline \multicolumn{2}{|c|}{ Code Minier Guinéen de 2011} & $\begin{array}{l}\text { Amendements au Code minier Guinéen de } \\
\qquad 2013\end{array}$ \\
\hline TVA & $\begin{array}{l}\mathrm{n} / \mathrm{a} \text { en phase de } \\
\text { construction ou d'ins- } \\
\text { tallation à condition } \\
\text { que les équipements } \\
\text { importés figurent sur la } \\
\text { liste minière. }\end{array}$ & $\begin{array}{l}\text { n/a en phase de construction ou d'installation } \\
\text { à condition que les biens importés figurent } \\
\text { sur la liste minière agréée avant le démarrage } \\
\text { des travaux par les Ministres des Mines et des } \\
\text { Finances à l'exception de fuel lourd. }\end{array}$ \\
\hline $\begin{array}{l}\text { Impôt Minimum For- } \\
\text { faitaire }\end{array}$ & $\begin{array}{l}\text { a) } \mathrm{n} / \mathrm{a} \text { en phase de } \\
\text { construction ou } \\
\text { d'installation et; } \\
\text { b) pendant trois (3) } \\
\text { ans à dater de la } \\
\text { première produc- } \\
\text { tion. }\end{array}$ & $\begin{array}{l}\text { a) n/a en phase de construction et; } \\
\text { b) pendant trois (3) ans à dater de la pre- } \\
\text { mière production }\end{array}$ \\
\hline $\begin{array}{l}\text { Impôt sur les Bénéfices } \\
\text { Industriels et Commer- } \\
\text { ciaux ou Impôt sur les } \\
\text { Sociétés }\end{array}$ & $\begin{array}{l}\text { a) } \mathrm{n} / \mathrm{a} \text { en phase de } \\
\text { construction ou } \\
\text { d'installation; } \\
\text { b) en phase d'exploi- } \\
\text { tation, il est dû au } \\
\text { taux de droit com- } \\
\text { mun. }\end{array}$ & Perçu dans toutes les phases. \\
\hline
\end{tabular}




\begin{tabular}{|c|c|c|}
\hline $\begin{array}{l}\text { Contribution des pa- } \\
\text { tentes }\end{array}$ & $\begin{array}{l}\text { a) } \mathrm{n} / \mathrm{a} \text { en phase de } \\
\text { construction ou } \\
\text { d'installation; } \\
\text { b) due en phase d'ex- } \\
\text { ploitation. }\end{array}$ & $\begin{array}{l}\text { a) n/a en phase de construction. } \\
\text { b) Due pendant la phase d'exploitation. }\end{array}$ \\
\hline $\begin{array}{l}\text { Contribution à la forma- } \\
\text { tion professionnelle } \\
\text { (CFP) }\end{array}$ & $\begin{array}{l}\text { a) n/a en phase de } \\
\text { construction ou } \\
\text { d'installation; } \\
\text { b) n/a pendant trois } \\
\text { (3) ans à dater de } \\
\text { la première pro- } \\
\text { duction en phase } \\
\text { d'exploitation. }\end{array}$ & $\begin{array}{l}\text { a) n/a en phase de construction ou d'instal- } \\
\text { latio. } \\
\text { b) pendant la phase d'exploitation, elle est } \\
\text { due }\end{array}$ \\
\hline $\begin{array}{l}\text { Droits d'enregistrement } \\
\text { et de timbre }\end{array}$ & $\begin{array}{l}\mathrm{n} / \mathrm{a} \text { en phase de } \\
\text { construction. }\end{array}$ & $\begin{array}{l}\text { Dû dans toutes les phases (voir code des im- } \\
\text { pôts) }\end{array}$ \\
\hline $\begin{array}{l}\text { Contribution Foncière } \\
\text { Unique (CFU) }\end{array}$ & $\begin{array}{l}\text { a) } \mathrm{n} / \mathrm{a} \text { en phase de } \\
\text { construction ou } \\
\text { d'installation et; } \\
\text { b) pendant trois (3) } \\
\text { ans à dater de la } \\
\text { première produc- } \\
\text { tion. }\end{array}$ & $\begin{array}{l}\text { a) n/a en phase de construction ou d'instal- } \\
\text { lation et; } \\
\text { b) pendant la phase d'exploitation au taux } \\
\text { de } 10 \% \text { pendant trois (3) à dater de la } \\
\text { première production, passé ce délai; le } \\
\text { ministre prendra les mesures pour sa per- } \\
\text { ception. }\end{array}$ \\
\hline $\begin{array}{l}\text { Impôt sur le Revenu } \\
\text { des Valeurs Mobilières } \\
\text { (IRVM) }\end{array}$ & $\begin{array}{l}\text { n/a en phase de } \\
\text { construction. }\end{array}$ & Dû dans toutes les phases. \\
\hline Taxe d'apprentissage & $\begin{array}{l}\text { a) } \mathrm{n} / \mathrm{a} \text { en phase de } \\
\text { construction ou } \\
\text { d'installation et; } \\
\text { b) pendant trois (3) } \\
\text { ans à dater de la } \\
\text { première produc- } \\
\text { tion. }\end{array}$ & $\begin{array}{l}\text { a) n/a en phase de construction } \\
\text { b) Due pendant la phase d'exploitation }\end{array}$ \\
\hline Stabilisation du régime & $\begin{array}{l}\text { 10ans avec possibilité } \\
\text { d'extension de } 5 \text { ans } \\
\text { contre prime annuelle }\end{array}$ & 15ans à dater de l'octroi du titre \\
\hline
\end{tabular}

